\title{
Green Software Services: From Requirements to Business Models
}

\author{
Schahram Dustdar, Fei Li, Hong-Linh Truong, Sanjin Sehic, \\ Stefan Nastic, Soheil Qanbari, Michael Vögler and Markus Claeßens \\ Distributed Systems Group \\ Vienna University of Technology, Austria \\ Email: lastname@dsg.tuwien.ac.at
}

\begin{abstract}
In recent years, green software research is gaining momentum because of the acute need for sustainable development. Most past research has been focused on the definitions, metrics and technical solutions for green software, but few has addressed green software from the business perspective. In this paper, we present the analysis on three key elements of Green Software Services (GSS)—-stakeholders, their requirements, and business models. The stakeholders of GSS are detailed with the services each stakeholder can provide and consume, thus clarifying their interests to GSS. Based on this analysis, we present the domain-independent, high-level requirements to GSS that cover diverse needs of different stakeholders. Six business models are then proposed to promote collaborations of stakeholders on the delivery of GSS. In the end, the relationship between GSS and cloud is discussed and a GSS marketplace is envisioned.
\end{abstract}

\section{INTRODUCTION}

In recent years, green software research is gaining momentum from the acute need for sustainable development as well as the far-reaching effect of ICT to our society. "[Green and] Sustainable Software is software, whose direct and indirect negative impacts on economy, society, human beings, and environment that result from development, deployment, and usage of the software are minimal and/or which have a positive effect on sustainable development."-Dick et al. [1]. Based on this definition, the green software research is growing in two directions. The first direction looks into the runtime energy consumption of software [2] and its engineering process [1]. The second looks into the important role that software plays in all conceivable aspects of our society, and investigate how software can be used to improve the sustainability of a broader range of business, social and individual activities [3]. This paper is focused on the research and development in the second direction-to leverage software to solve sustainability problems in a broader scope.

The emergence of cloud computing and Internet of Things makes software services further reach out to the physical environments at a larger scale. Many existing business operations are being improved in the respects of scalability, automaticity, manageability and so on. Such trend has huge implications to green software research since sustainability can be improved by applying software services based on these new computing paradigms. This view prompts us to rethink the business models of green software: when green software is delivered as online services, or Green Software
Services (GSS), a broader range of business, governments and individual processes can more easily employ the services to reduce their energy consumption. Furthermore, more flexible business relationships can be established between different stakeholders, so that the financial and social interests of green software can in turn promote its research and development.

To this end, this paper presents our analysis from the business perspective of GSS. The research is aimed at providing a systematic, high-level view on the three key elements that motivate the development of GSS: stakeholders, their requirements, and business models. The stakeholders of GSS are detailed with the services they can provide and consume, thus clarifying their interests to GSS. Based on this analysis, we present the domain-independent core requirements to GSS that are concerned by different stakeholders. Six business models are then proposed to promote collaborations of stakeholders on the delivery of GSS. The business models are based on the stakeholder analysis as well as our experiences in applying GSS to various business domains, such as smart buildings and smart transportation. In the end, we will discuss how the business models are related to cloud services and the challenges of realizing a marketplace of GSS.

The remaining part of this paper is structured as follows: Section II introduces the core stakeholders and their relationships in GSS. Then the requirements for GSS are analyzed in Section III. Section IV presents the business models in detail. Finally, Section V discusses the implications of these business models to the research and development of GSS and concludes the paper.

\section{STAKEhOLDERS IN GSS}

In order to investigate the requirements for GSS, we first conduct a detailed analysis on the related stakeholders. Overall, the stakeholders are classified to core stakeholders and supportive stakeholders. Core stakeholders are those business entities that have direct business interests in providing GSS, whereas supportive stakeholders are those organizations or individuals who have financial or social interests to GSS but not directly gain profit from delivering GSS. The stakeholders are illustrated in Figure 1.

\section{1) Core stakeholders:}

- Business service providers are operating diverse businesses that might benefit from GSS, e.g. building op- 


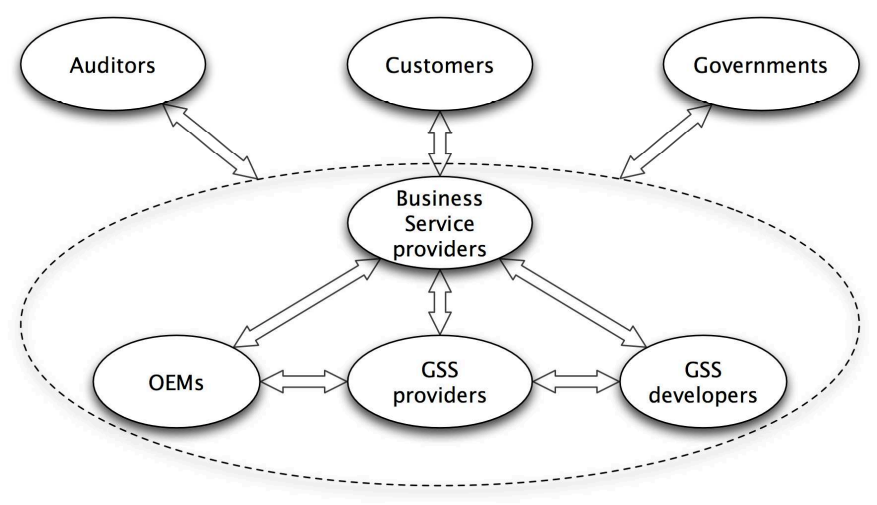

Fig. 1. Stakeholders of GSS.

erators, transportation services, data centers. They share one common objective of maximizing the sustainability of their businesses (by saving energy or reducing wastes). Since they have the direct financial incentives of reducing operational costs by applying GSS to their existing businesses, they are the main driving force for the development of GSS.

- OEMs produce equipment that are the source of energy consumption. Their efforts alone on developing sustainable equipment can result in significant energy conservation (e.g. LED lights and energy-efficient chillers). Integrating, managing and leveraging the energy saving capabilities of OEM devices are one of the most important approaches of realizing GSS. More importantly, GSS is able to optimize complex systems that constitute a large number of OEM equipment [4].

- GSS providers provide GSS that are used by business service providers. The services are in diverse business domains and of various functions, such as home automation, facility management, offline analysis and so on. The services provided by them can be realized by other stakeholders or domain experts. GSS providers retain the service interface and establish direct business relationships with customers who need GSS.

- GSS developers realize business logics and optimization methods in the target domain. Different implementations of the same business logic could have considerably different effects in terms of sustainability. Thus domain knowledge is often required for GSS developers. Promoting a GSS developer community will help to leverage the growing amount of data available on the internet [5] and the increasingly connected devices [6] to create more diverse GSS and applying them to more business domains.

2) Supportive stakeholders:

- Governments are ultimately responsible for promoting sustainable development. Their activities such as policy making, policy enforcement, legislation and standards enactment are essential for the adoption and long-term growth of GSS. Governments are also important public information providers.

- Auditors systematically assess the performance of GSS. They provide a solid baseline for comparing and further improving GSS by applying standardized evaluation methods. Audit may be apply to any system components or domain-specific business services.

- Service consumers are, in most current GSS applications, passive stakeholders who benefit from business services at reduced costs. However, since the behavior of consumers are also decisive to the effects of GSS, the usage information and behavior patterns can be collected for designing better GSS.

Table I summarizes the main services provided and consumed respectively by each stakeholder.

\section{REQUIREMENTS FOR GSS}

Based on the previous analysis about different stakeholders and their involvement in GSS, this section presents the highlevel requirements to GSS. These requirements are intended to be domain independent, with each of them addresses the needs of multiple stakeholders.

1) Identifying core services (Concerned stakeholders: All core stakeholders)

Given our perspective that green software is software designed to improve the sustainability of other business, social or individual activities, easily engaging with various target domains is the key to the wide adoption of GSS. This means to identify a set of core GSS capabilities that are independent from the domain specifics of target systems, while making GSS easily adaptable to address domain requirements. Therefore, several core capabilities expected from GSS are as follows.

- Collecting and preparing data from target systems. This means that GSS should have the capability to access and acquire raw data from diverse environments that are to be made "greener", including physical environments, hardware, software and information generated by humans. Thus GSS should not be restricted to certain communication protocols or data exchange formats. Furthermore, for the data to be effectively utilized in GSS, GSS has to prepare the raw data at two levels. The basic level is syntactic preparation to normalize the presentation of the data. This task is covered in many domain-specific standardization efforts like oBIX (Open Building Information Exchange) [7]. The higher level of data preparation is in the ongoing research on semantic technologies, which aims at the semantic interoperability of systems [8].

- Customizing for different target systems. Providing the GSS to a specific target system means that GSS should be tailored on provisioning. Such tailoring can either be physically separating system components, configuring them and deploying only the necessary components for the target system, or 


\begin{tabular}{|c|c|c|}
\hline Stakeholders & Services or information provided & Services or information consumed \\
\hline Business service providers & $\begin{array}{l}\text { - Domain-specific business service } \\
\text { - Domain process optimization knowledge }\end{array}$ & $\begin{array}{l}\text { - Optimization services } \\
\text { - Auditing }\end{array}$ \\
\hline GSS providers & $\begin{array}{l}\text { - Device integration } \\
\text { - Optimization services } \\
\text { - Data acquisition } \\
\text { - Analytics } \\
\text { - Data visualization } \\
\end{array}$ & $\begin{array}{l}\text { - Domain-specific knowledge } \\
\text { - Device connectivity } \\
\text { - Application development }\end{array}$ \\
\hline OEMs & $\begin{array}{l}\text { - Devices } \\
\text { - Device optimization knowledge }\end{array}$ & - Device integration \\
\hline Application developers & $\begin{array}{l}\text { - Business logic implementation } \\
\text { - Optimization methods implementations }\end{array}$ & $\begin{array}{l}\text { - GSS platform services } \\
\text { - Domain-specific knowledge } \\
\text { - Device optimization knowledge }\end{array}$ \\
\hline Governments & $\begin{array}{l}\text { - Raising public awareness to GSS } \\
\text { - Public information } \\
\text { - Regulation and legislation } \\
\text { - Policy enforcement }\end{array}$ & $\begin{array}{l}\text { - Standardization } \\
\text { - Auditing results }\end{array}$ \\
\hline Auditors & - Auditing & $\begin{array}{l}\text { - Data access } \\
\text { - Process monitoring }\end{array}$ \\
\hline Service consumers & - Usage feedbacks & $\begin{array}{l}\text { - GSS } \\
\text { - Business services }\end{array}$ \\
\hline
\end{tabular}

ROLES OF STAKEHOLDERS

virtually excluding the target system from using other irrelevant system capabilities. Thus extensibility and customizability are critical to GSS.

- Accommodating various scales. The target systems of GSS may be of largely different scales, ranging from a single home to a large city [9]. They differ with each other in terms of numbers and types of equipment, data volume, and resource requirements for applications. Thus GSS should be customized not only for specific functions, but also customized in terms of the resources needed for each target system. This requires flexible allocation of computing resources and on-demand scaling of GSS.

These core capabilities form the basic features for a domain-independent platform that can serve as the basis of green software services.

2) Supporting a broad range of process optimization and analytics methods (Concerned stakeholders: GSS providers, Business service providers, GSS developers) GSS is not limited to a certain optimization method, but has to decide on the exact method to be used according to the specific task at hand. The known methods for optimizing energy usage include offline data modeling and simulation [4][10], context-aware controls (e.g. presence-based light control), agent-based systems [11]. The growing amount of data further points GSS to employ the results in big data research. The implication of this requirement is that the optimization capability of GSS lies in the capabilities of handling various types of data formats, including time-series data, well-structured data, unstructured data or even natural language.

3) Supporting realization and enforcement of sustainability policies (Concerned stakeholders: GSS providers, Business service providers, Governments, Auditors)

Supporting sustainability policies is one of the basic expectations to GSS. Multiple aspects need to be considered for the realization of sustainability policies. First of all, GSS is required to model and understand the sustainability policies of the target systems, e.g. temperature limit for a green building. Second, GSS is required to find efficient ways of meeting the goals defined by the policies, e.g. how to efficiently control the HVAC systems. Third, in realizing the policies, GSS themselves should be energy-efficient, complying to sustainability policies for IT systems.

4) Ensuring privacy and security (Concerned stakeholders: Business service providers, Service consumers)

Given the sensitivity to private, commercial and public data that might be used in GSS, data privacy, security and confidentiality have to be incorporated into GSS as first-class citizens from the beginning and visible during every stage of development of the system. Furthermore, since GSS usually need to apply certain controls or changes in order to change the energy consumption of target systems, such control capabilities are to be secured so that only the authorized software components and personnel can perform the allowed controls.

5) Supporting collaborations between stakeholders (Concerned stakeholders: All stakeholders)

As stated in the stakeholder analysis, successful GSS are not built by any single party, but built by the collaborations of multiple stakeholders, each of which provides their knowledge and services. The collaborations are the basis for flexible business models. Thus GSS need to support collaborations by providing interfaces for different stakeholders, e.g. interfaces for thirdparty developers to develop new functions or interface for auditors to inspect the sustainability status. More importantly, such collaborations are not only reflected on system interfaces, but also the capability of directly 
presenting and sharing business interests on serving certain needs of GSS stakeholders.

\section{Business MODELS}

The GSS platforms that fulfill the requirements will become the cornerstone of flexible GSS business models.

\section{A. Description approach}

This section uses an established Business Model Framework [12] to describe the business models of GSS. The framework is illustrated in Figure 2.

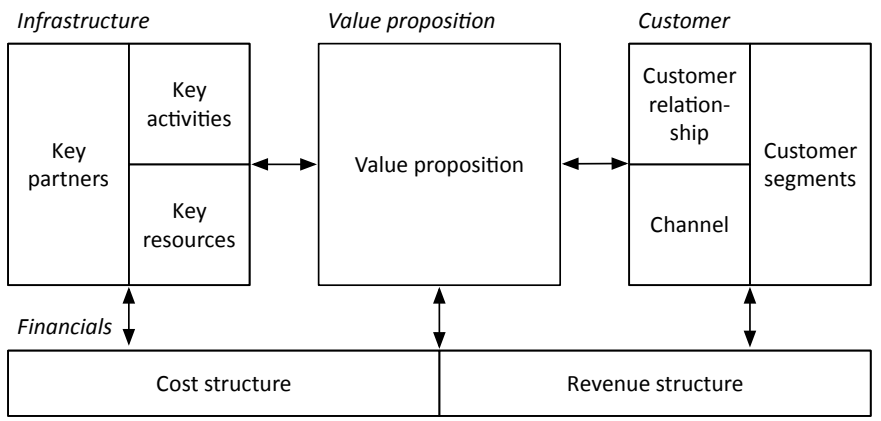

Fig. 2. The method of describing business models

A business model is described in four areas-Financials, Infrastructure, Customer and Value Proposition. The cost structure in Financials area is directly related to the stakeholders who are providing resources and conducting service activities, whereas the revenue structure in Financials area is customers who are interested in the specific services. Overall, the three area converge on the value proposition of a business model. Readers can refer to Bucherer et al. [12] to find more details on the description framework.

\section{B. Business models}

1) Infrastructure services: provide software services for accessing the optimization capabilities on OEM devices directly, illustrated in Figure 3.

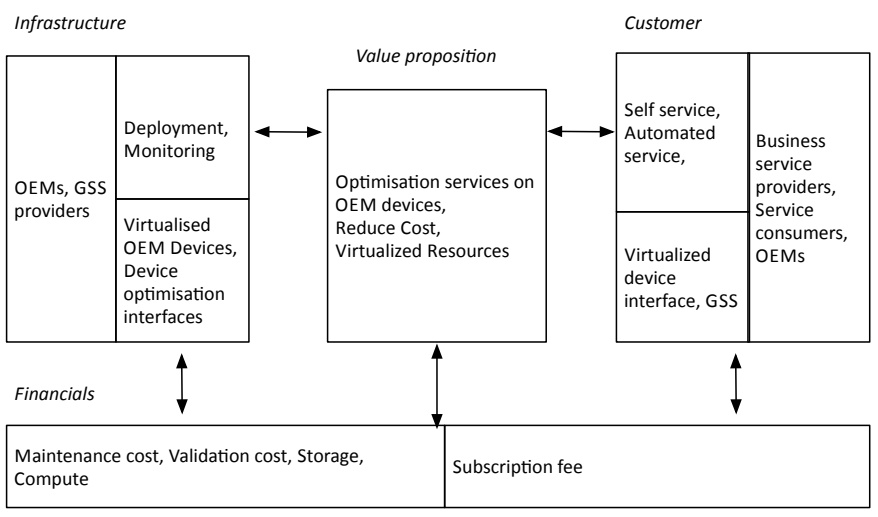

Fig. 3. Infrastructure services

Its core value proposition is to provide optimization services on OEM devices, thus directly reducing the cost of using these devices. OEM devices are virtualized [13] as resources so that GSS can easily access and control them. GSS providers are responsible for device virtualization that opens up the optimization interfaces. The customer value that can be created by this model is mainly to provide business service providers and their service consumers the opportunity to efficiently operate their facilities, without requesting the support of OEMs. OEMs also benefit from this model through improved automation on their customer services since maintenance activities can be automated. The services under this model can be charged by service subscription.

2) Platform services: are the most comprehensive and flexible business models, illustrated in Figure 4.

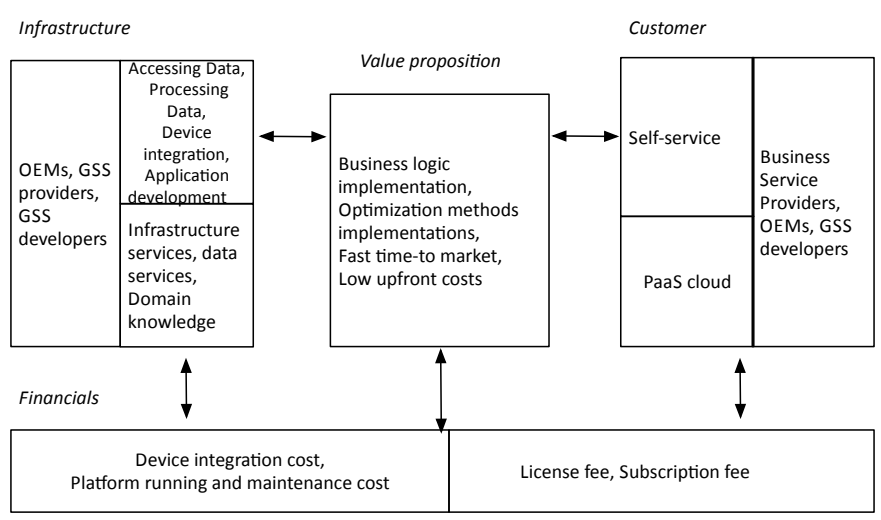

Fig. 4. Platform services

It is not limited to certain business domains or optimization methods. Alternatively, it aims at providing services such as device integration, data access, data processing, analytics libraries on a common platform. OEMs can use the platform to realize the first business model-infrastructure services. GSS developers can develop GSS for specific customers on top of the platform, and GSS providers can operate the platform. The platform provides the core capabilities discussed in the requirements section. Stakeholders can use the platform through a self-service portal. Essentially, this model is an adaptation of PaaS cloud for GSS. For stakeholders using the platform, the key value proposition is to realize green business logics and domain-specific optimization methods at lower upfront cost with faster time-to-market.

3) Virtual verticals: are provided to vertical applications such as smart buildings or data centers, illustrated in Figure 5.

"Vertical" means that such applications are delivered as an end-to-end service including physical devices, middleware and applications for a certain physical environment. Virtual verticals can be realized by the collaborations of GSS providers, OEMs and GSS developers. They integrate physical devices in the target environment and develop dedicated applications, such as light control or chiller management. For business service providers, who are the direct customers of virtual verticals, they enjoy their dedicated GSS operational environments without committing computing resources or maintaining an IT platform for GSS. Instead, they subscribe to virtual vertical 


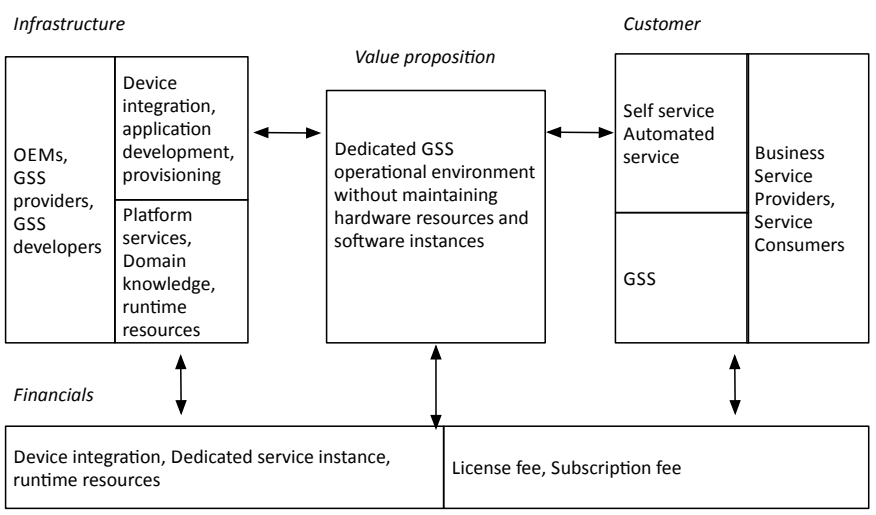

Fig. 5. Virtual verticals

services or license the software services for their operational environment.

4) Data services: The amount of data generated by various business services is rapidly growing. When the confidentiality of data is properly managed, providing data to external experts or the public will further increase the utility of data. The business model is illustrated in Figure 6.

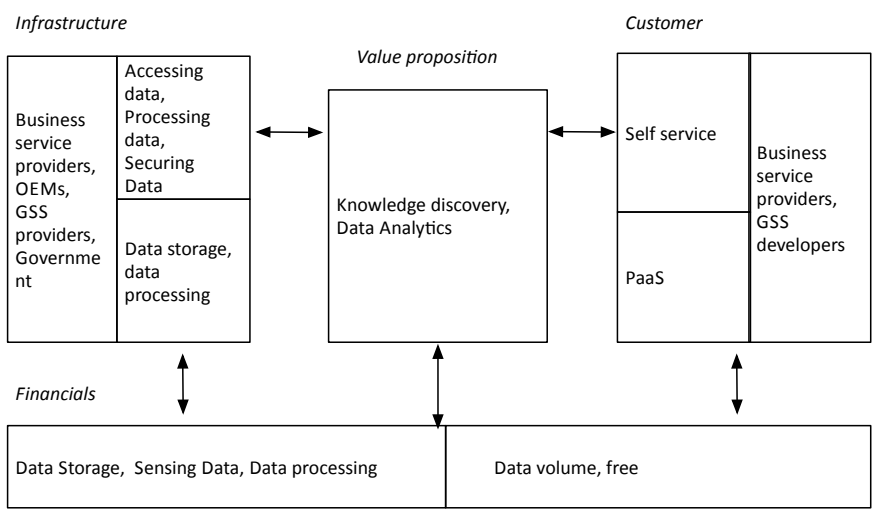

Fig. 6. Data services

The data are owned by business service providers, OEMs or governments. GSS providers offer the platform for them to open data access and establish business relationships with the customers who are interested in using the data for knowledge discovery or analytics. Business service providers can benefit from publicly available data to optimize their operations. GSS developers can create novel applications on the data, or discover hidden knowledge in the data. The idea of providing data services have been realized through platforms like Cosm [5], and more public data are being made available [14] for developer community to discover their value.

5) Third-party applications: When the access to data, infrastructure and core services are open to third-party developers, novel applications can be developed and provided as GSS, illustrated in Figure 7.

This business model is built on top of infrastructure services, platform services and data services. The range of applica-

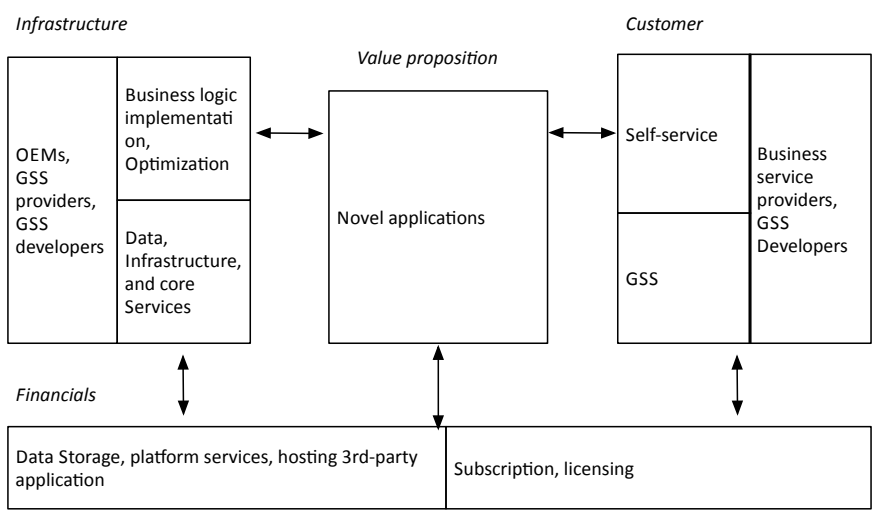

Fig. 7. Third-party applications

tions can be broad, including for example optimized business processes, device optimization methods or analytics. On GSS platform, the applications are offered online and used through subscription or licensing. The GSS platform is responsible for the provisioning of resources to run the applications.

6) Analytics as a services: Analytics on the sustainability of business services are delivered by third-party analytics services [15], illustrated in Figure 8.

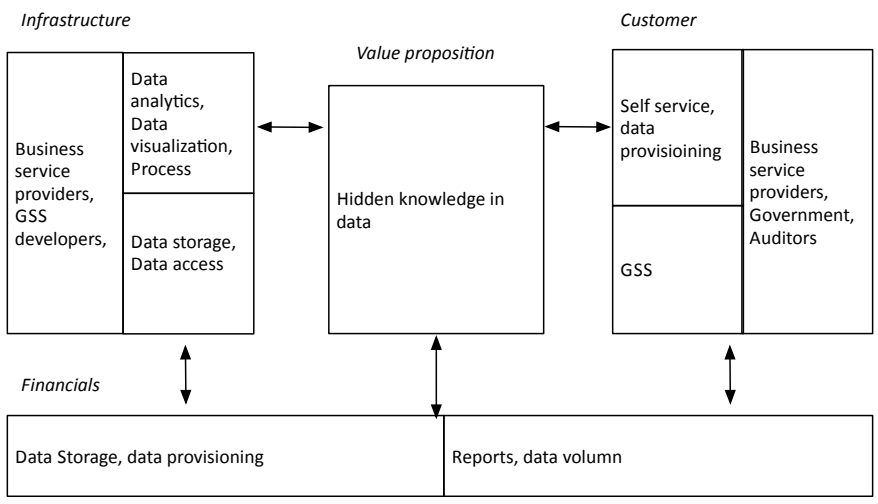

Fig. 8. Analytics as a services

This model is to a certain extent a type of third-party applications. However, analytics as a service stresses that analytics are highly specialized tasks. External experts specialized on analytics are often required for conducting offline analysis. This model can also be employed by external auditing in order to assess the performance of GSS. For the data to be effectively used in analytics, provisioning [16] (e.g. cleansing, normalization) is important, but not necessarily a task of data providers since the provisioning can also be handled by data experts. Business service providers, governments and auditors can all be interested in the results of analytics. They may purchase the reports or pay by the volume of data being analyzed. 


\section{DISCUSSIONS AND CONCLUSION}

\section{A. A cloud perspective to GSS}

The rapidly growing popularity of cloud computing has made energy consumption of large data centers a trending topic in cloud research. Both of the two perspectives of green software research-energy efficiency of software and using software for energy efficiency-are applicable to cloud. On the one hand, the energy efficiency of cloud is affected by the operating systems, middleware and applications. On the other hand, software tools can be built in order to manage the energy consumption of cloud. Mechanisms like resource scheduling and application workload prediction have been widely applied and are still improving.

This paper relates cloud and green software in a new perspective: the service models of cloud. IaaS (Infrastructure as a Service), PaaS (Platform as a Service) and SaaS (Software as a Service) [17] are already familiar to researchers and IT professionals. When green software services are provided on the Internet, these three models are referred in order to classify the services provided by each stakeholder. In fact, the business models proposed in this paper can easily be mapped to the cloud service models. Infrastructure services that open up interfaces of OEM devices are similar to the IaaS cloud, which provides computing resources as services. The platform services and data services can be realized on a PaS cloud by extending its capabilities of integrating with OEM devices. Third-party applications, Virtual verticals and Analytics-as-aService are different forms of SaaS. The result of such analogy prompts us to conceive a cloud-based green software platform that fulfills the requirements outlined in Section III, so that the stakeholders can collaborate based on different business models and create novel green software services.

\section{B. Towards a marketplace of GSS}

The core vision of this paper is to promote stakeholders in GSS to establish flexible business relationships. A natural result of growing participation of stakeholders is that when the number of stakeholders grow, a marketplace will emerge. In the marketplace, each type of service could be provided by multiple stakeholders, who offer different implementations for the same service with different QoS and price. Therefore, on top of the common platforms of GSS that stakeholders can collaborate at a technical level, there is a further need of supporting business activities including for example billing and SLA monitoring.

Although a cloud-based marketplace has been demonstrated by Amazon ${ }^{1}$ and the concept of application store ${ }^{2}$ is well accepted, a marketplace for GSS will face several new challenges. Evaluating and comparing GSS are hard since the effect of each service to each customer is tightly related to the specifics of target systems and their physical environments. Comprehensive metrics, especially domain-specific metrics,

\footnotetext{
${ }^{1}$ https://aws.amazon.com/marketplace

${ }^{2}$ https://play.google.com/store
}

need to be incorporated or developed for objectively and accurately describing the services in the marketplace. These metrics will also help to establish effective monitoring mechanisms for GSS. A marketplace for GSS also means that the services should be delivered online. This challenges the deployment and provisioning mechanisms for GSS since business models such as Infrastructure services and Virtual verticals need to be coupled with devices in customers' physical environments. Automated or semi-automated methods, such as device integration or customer tools, need to be created to enable efficient and customized service delivery.

\section{Conclusion}

The research on the novel topic of green software is still at its infancy. Early research problems and technical solutions have been proposed, but wide adoption of green software is yet to happen. In this paper, we tackled green software from the business perspective-trying to identify the stakeholders and the business models that could benefit different stakeholders. We detailed the stakeholders and their interests to GSS. Based on this analysis, the high-level requirements to GSS were identified. Diverse business models were then proposed in order to motivate stakeholders to collaborate on the delivery of GSS. In the end, we discussed how the business models are related to cloud service models and the challenges of realizing a marketplace for GSS.

\section{ACKNOWLEDGMENT}

This work is sponsored by Pacific Controls Cloud Computing $\mathrm{Lab}\left(\mathrm{PC}^{3} \mathrm{~L}\right)^{3}$, a joint lab between Pacific Controls L.L.C., Dubai and the Distributed Systems Group of the Vienna University of Technology.

\section{REFERENCES}

[1] M. Dick, S. Naumann, and N. Kuhn, "A Model and Selected Instances of Green and Sustainable Software," in What Kind of Information Society? Governance, Virtuality, Surveillance, Sustainability, Resilience SE - 24, ser. IFIP Advances in Information and Communication Technology, J. Berleur, M. Hercheui, and L. Hilty, Eds. Springer Berlin Heidelberg, 2010, vol. 328, pp. 248-259.

[2] B. Steigerwald and A. Agrawal, "Developing Green Software," Intel, Tech. Rep., 2011.

[3] S. Dustdar, C. Dorn, F. Li, L. Baresi, G. Cabri, C. Pautasso, and F. Zambonelli, "A roadmap towards sustainable self-aware service systems." Cape Town, South Africa: ACM, 2010, pp. 10-19. [Online]. Available: http://portal.acm.org/citation.cfm?id=1808984.1808986

[4] J. Cook, D. Smith, and A. Meier, "Coordinating Fault Detection, Alarm Management, and Energy Efficiency in a Large Corporate Campus," in 2012 ACEEE Summer Study on Energy Efficiency in Buildings, 2012, pp. 83-93.

[5] Cosm, "Cosm-Internet of Things Platform, Connecting Devices and Apps for Real-time Control and Data Storage."

[6] L. Atzori, A. Iera, and G. Morabito, "The Internet of Things: A survey," Computer Networks, vol. 54, no. 15, pp. 2787-2805, Oct. 2010. [Online]. Available: http://dl.acm.org/citation.cfm?id=1862461.1862541

[7] OASIS, "Open Building Information Exchange (oBIX)." [Online]. Available: https://www.oasisopen.org/committees/tc_home.php?wg_abbrev=obix

[8] N. Loutas, E. Kamateri, and K. Tarabanis, "A Semantic Interoperability Framework for Cloud Platform as a Service," in 2011 IEEE Third International Conference on Cloud Computing Technology and Science. IEEE, Nov. 2011, pp. 280-287.

\footnotetext{
${ }^{3}$ http://pc3l.infosys.tuwien.ac.at/
} 
[9] P. C. S. LLC., "Pacific Controls-Technology for Sustainable Development." [Online]. Available: http://www.pacificcontrols.net/projects/ictproject.html

[10] D. Zachhuber, J. Doppler, A. Ferscha, C. Klein, and J. Mitic, "Simulating the Potential Savings of Implicit Energy Management on a City Scale." IEEE Computer Society, 2008, pp. 207-216.

[11] G. James, D. Cohen, R. Dodier, G. Platt, and D. Palmer, "A deployed multi-agent framework for distributed energy applications." Hakodate, Japan: ACM, 2006, pp. 676-678. [Online]. Available: http://portal.acm.org/citation.cfm?id=1160752

[12] E. Bucherer and D. Uckelmann, "10 Business Models for the Internet of Things," Business, pp. 1-25, 2011.

[13] D. Guinard, V. Trifa, S. Karnouskos, P. Spiess, and D. Savio, "Interacting with the SOA-Based Internet of Things: Discovery, Query, Selection, and On-Demand Provisioning of Web Services," IEEE Transactions on Services Computing, vol. 3, no. 3, pp. 223-235, Jul. 2010.

[14] Greenbiz, "Hack City-Verge SF @ Greenbuild Resources," 2012.

[15] X. Sun, B. Gao, L. Fan, and W. An, "A Cost-Effective Approach to Delivering Analytics as a Service," in 2012 IEEE 19th International Conference on Web Services. IEEE, Jun. 2012, pp. 512-519.

[16] F. Li, S. Nastic, and S. Dustdar, "Data Quality Observation in Pervasive Environments," in The 10th IEEE/IFIP International Conference on Embedded and Ubiquitous Computing (EUC 2012), Paphos, Cyprus, 2012.

[17] F. Liu, J. Tong, J. Mao, R. Bohn, J. Messina, L. Badger, and D. Leaf, "NIST Cloud Computing Reference Architecture," NIST Special Publication, vol. 500, p. 292, 2011. 